\title{
Purification and Characterization of Insulin-Mimetic Inositol Phosphoglycan-Like Molecules From Grass Pea (Lathyrus sativus) Seeds
}

\author{
Covadonga Pañeda, ${ }^{1}$ Ana Victoria Villar, ${ }^{2}$ Alicia Alonso, ${ }^{2}$ Félix M. Goñi, ${ }^{2}$ Federico Varela, ${ }^{3}$ \\ Urs Brodbeck, ${ }^{4}$ Yolanda León, ${ }^{1}$ Isabel Varela-Nieto, ${ }^{1 *}$ and David R. Jones ${ }^{5 *}$ \\ ${ }^{1}$ Instituto de Investigaciones Biomédicas “Alberto Sols” (C.S.I.C.), Madrid, Spain \\ ${ }^{2}$ Unidad de Biofísica (C.S.I.C.-U.P.V./E.H.U.) and Departamento de Bioquímica, Universidad del Pais Vasco, \\ Bilbao, Spain \\ ${ }^{3}$ Centro de Recursos Fitogenéticos-Instituto Nacional de Investigación y Tecnología Agraria y Alimentaria \\ (C.R.F-I.N.I.A) Alcalá de Henares, Madrid, Spain \\ ${ }^{4}$ Institute for Biochemistry and Molecular Biology, University of Berne, Berne, Switzerland \\ ${ }^{5}$ Department of Immunology and Oncology, Centro Nacional de Biotecnología (C.S.I.C.), Cantoblanco, \\ Madrid, Spain
}

Accepted March 5, 2001

\begin{abstract}
Background: Signal transduction through the hydrolysis of glycosyl-phosphatidylinositol (GPI) leading to the release of the water-soluble inositol phosphoglycan (IPG) molecules has been demonstrated to be important for mediating some of the actions of insulin and insulin-like growth factor-I (IGF-I).

Materials and Methods: In the present study, GPI from grass pea (Lathyrus sativus) seeds has been purified and partially characterized on the basis of its chromatographic properties and its compositional analysis.

Results: The results indicate that it shows similarities to GPI previously isolated from other sources such as
\end{abstract}

rat liver. IPG was generated from $L$. sativus seed GPI by hydrolysis with a GPI-specific phospholipase D (GPI-PLD). This IPG inhibited protein kinase A (PKA) in an in vitro assay, caused cell proliferation in explanted cochleovestibular ganglia (CVG), and decreased 8-Br-cAMP-induced phosphoenolpyruvate carboxykinase (PEPCK) mRNA expression in cultured hepatoma cells.

Conclusions: Our data indicate that L. sativus seed IPG possess insulin-mimetic activities. This may explain why L. sativus seeds have been used in some traditional medicines to ameliorate diabetic symptoms.

\section{Introduction}

Glycosyl-phosphatidylinositol (GPI) lipids have a structural role as protein anchors to the outer cell surface $(1,2)$. In addition, they are implicated in hormone, growth factor, and cytokine signal transduction $(2,3)$. Their phosphodiesteric hydrolysis, mediated by an activated phospholipase, results in the generation of low-molecular-weight, watersoluble oligosaccharide species termed inositol phosphogylcans (IPGs). IPGs have been demonstrated to possess biological properties when added exogenously to cells, in many cases mimicking the biological effects of hormones and growth factors such as insulin and insulin-like growth factor I (IGF-I) $(3,4)$. Distinct IPG species have been purified and characterized from a variety of animal sources. The best characterized of these is that from rat liver $(2,3)$. Despite the relative ease of IPG purification, a suitable biological source of

Address correspondence and reprint requests to: Isabel VarelaNieto, Instituto de Investigaciones Biomédicas "Alberto Sols" (C.S.I.C.), Arturo Duperier 4, 28029 Madrid, Spain. Phone: +34-91-585 4608; Fax: +34-91-585 4587; e-mail: ivarela@iib.uam.es *I. Varela-Nieto and D.R. Jones contributed equally to this work.
IPG is still to be found that would comply with the following criteria: (i) high yield of biologically active IPG, (ii) low cost of purification, and (iii) abundance of starting material. These three criteria are considered essential for the large-scale preparation of IPG species.

Diabetes is a chronic and presently incurable disease that affects an ever-growing proportion of the world's population. In spite of the modern advances in drug design to alleviate and delay the symptoms of diabetes, there still remains some interest in the use of nonregistered concoctions that originate from plant origin. Such plants including Medicago sativa (5), Ginkgo biloba (6,7), Bryonia alba $(8,9)$, Burmelia sartorum $(10)$, and others $(11,12)$ have been considered to have "anti-diabetic properties." The preparation of extracts from these plants has been poorly documented and one would envisage such preparations would be subject to significant variation depending both on geographic location and the method used. Some of the compounds within these preparations might be putative IPG molecules. However, all these reports lack compelling evidence regarding the exact chemical composition, structure, and mechanism of action of the 
preparations. Grass pea (Lathyrus sativus) is a part of traditional culture in rural Spain. Eating L. sativus seeds has been a popular remedy to improve the condition of diabetic patients and as a general tonic in Andalusia and the Canary Islands (13). On the basis of our experience both in the area of IPG purification and assessment of its biological properties and the above considerations regarding the search for a suitable source of IPG, we started this study to determine the presence of IPG in L. sativus seeds with the objective of characterizing for the first time plant-derived IPG-like molecules.

\section{Materials and Methods Materials}

L. sativus seeds were from the C.R.F.-I.N.I.A. collection (Madrid, Spain). $\left[\gamma^{32} \mathrm{P}\right]$ ATP and $\left[{ }^{32} \mathrm{P}\right] \mathrm{dCTP}$ (specific activities $3000 \mathrm{Ci} / \mathrm{mmol}$ ) were purchased from Little Chalfont, Amersham, UK. Fetal bovine serum (FBS), cell culture media, and supplements were purchased from GibcoBRL (Paisley, UK). Silica gel thin layer chromatography (t.l.c.) plates were purchased from Merck (Darmstadt, Germany). Authentic lipid standards, histone IIA, PKA, orcinol, and the molybdenum blue reagent were purchased from Sigma (Poole, UK). A murine monoclonal antiproliferative cell nuclear antigen (PCNA) antibody was obtained from ATOM (Barcelona, Spain). Phosphatidylinositol-specific phospholipase C (PI-PLC), from Bacillus cereus, was purchased from Molecular Probes (Leiden, The Netherlands). GPI-specific phospholipase C (GPI-PLC), from Trypanosoma brucei, was bought from Oxford Glycosystems (Oxford, UK). GPI-specific phospholipase D (GPI-PLD) was purified from bovine serum as reported (14). Other reagents were of analytic grade or better and were from Merck.

\section{Methods}

PURIFICATION OF L. sativus SEED GPI. GPI Was purified as detailed (15). Briefly, total lipids isolated by $\mathrm{CHCl}_{3} / \mathrm{MeOH} / \mathrm{HCl}$ extraction of $50 \mathrm{~g}$ of L. sativus seeds were adsorbed onto silica G60. The contaminants were removed first with chloroform and then the pellet from a $2800 \mathrm{~g}$ spin was washed three times in a chloroform:methanol: $\mathrm{HCl}$ (300:50:3) solution. Polar lipids were extracted by washing the silica with methanol. This extract was applied to the origin of heat-activated t.l.c plates. The plates were then developed twice in the acidic solvent consisting of $\mathrm{CHCl}_{3} / \mathrm{CH}_{3} \mathrm{COCH}_{3} / \mathrm{MeOH} / \mathrm{CH}_{3} \mathrm{COOH} /$ $\mathrm{H}_{2} \mathrm{O}$ (10/4/2/2/1, by volume). The material remaining at the origin (from $-0.5 \mathrm{~cm}$ below to $+1 \mathrm{~cm}$ above) was eluted from silica by washing three times with methanol. The eluted material was dried under a gentle steam of nitrogen gas before it was applied to a further set of t.l.c. plates for development in the basic solvent consisting of $\mathrm{CHCl}_{3}$ / $\mathrm{MeOH} / 16 \mathrm{M} \mathrm{NH} \mathrm{NH}_{3} / \mathrm{H}_{2} \mathrm{O}$ (45/45/3.5/10, by volume).
GPI comigrated with a rat liver GPI standard and was located between the authentic standards phosphatidic acid (PtdOH) and phosphatidylcholine (PtdCho). GPI was eluted from silica by washing three times with methanol. The eluted material was passed through a $0.22-\mu \mathrm{m}$ nylon filter to remove silica before drying under a gentle steam of $\mathrm{N}_{2}$. GPI was stored dry under $\mathrm{N}_{2}$ at $-80^{\circ} \mathrm{C}$. Confirmation that GPI was indeed the lipid migrating at the stated position was achieved by noting its reaction with $\mathrm{I}_{2}$ vapor, Coomassie blue (both general lipid stains), ninhydrin (a stain for free amino groups), molybdenum blue reagent (a stain for phospholipid phosphate), and orcinol/sulphuric acid (a stain for sugar residues). Free amino and phosphate groups within the eluted GPI were estimated as previously described (15). The stoichiometry of sugar residues within GPI was determined using orcinol/ sulphuric acid with reference to authentic synthetic IPG analog standards $(16,17)$. To determine the fatty acid composition of the purified GPI, gas chromatography-mass spectrometry (GC/MS) was used as previously described (18). GPI was also purified from rat liver (15).

HYDROLYTIC SUSCEPTIBILITY OF L. sativus SEED GPI BY Phospholipases. Purified L. sativus seed GPI was reconstituted asymmetrically into preformed liposomal membranes according to previously published methods $(18,19)$. GPI-PLD, GPI-PLC, and PI-PLC were employed in the incubations. The specificity of the enzymes has been reported (18-20). The percentage of GPI hydrolyzed was determined as previously described $(18,19)$. The upper aqueousmethanolic phase formed after termination of the GPI hydrolysis incubation with GPI-PLD was lyophilized. The amount of IPG generated was determined by measuring the inorganic phosphate and amino groups within this fraction $(18,19)$. The percentage of GPI hydrolysis was approximately $90 \%$. IPG was filter sterilized into Hank's salts solution for testing biological activity. Until use, it was either stored lyophilized or in solution at $-80^{\circ} \mathrm{C}$. Rat liver IPG (rIPG) was also prepared, quantified, and stored using the above method.

BIOLOGICAL ASSAYS. The ability of the IPG to inhibit the activity of protein kinase A (PKA) was assessed by using a previously published method (21). Explanted cochleovestibular ganglia (CVG) were isolated from chicken embryos (embryonic day 3) as described (22). Organotypic cultures of CVG were treated with IPG and rIPG at different concentrations as previously described (23). IPG-induced cell proliferation was assessed using two methods: by morphometric analysis of the CVG after 24-hr treatment with IPG and by the expression of the PCNA, a 36-kDa nuclear protein essential for DNA synthesis and cell-cycle expression, by Western blotting (a 1:500 dilution was used) as previously 
described (24-26). A secondary antibody conjugated to peroxidase was purchased from Bio-Rad (Hercules, CA, USA) and was used according to the supplier.

\section{RNA Extraction and Northern Blot Analysis of PEPCK}

This followed a previously published method (27). Briefly, H35 cells were routinely grown in Dulbecco's Modified Eagles Medium with 10\% FBS, $2 \mathrm{mM}$ of L-glutamine, $100 \mathrm{U} / \mathrm{ml}$ of penicillin, and $100 \mu \mathrm{g} / \mathrm{ml}$ of streptomycin at $37^{\circ} \mathrm{C}$ in a watersaturated atmosphere containing $5 \% \mathrm{CO}_{2}$. After $24 \mathrm{hr}$ of serum starvation, cultures of $\mathrm{H} 35$ cells were incubated in the presence of 8-Br-cAMP for $5 \mathrm{hr}$. Saline (control), insulin, rIPG, or IPG were added to the medium for the last $2 \mathrm{hr}$ of 8 -Br-cAMP treatment. Total H35 cell RNA was isolated by the guanidinium-thiocianate-phenol-chloroform extraction method (28). Total RNA (20 $\mu$ g per sample) was size fractionated by electrophoresis in a $1.2 \%$ agarose gel under denaturing conditions (1.1 M of formaldehyde in MOPS buffer) and transferred to nitrocellulose filters (Schleicher and Shuell, Dassel, Germany). Prehybridization and hybridization of membranes were performed under moderate stringency conditions $\left(50 \%\right.$ formamide at $42^{\circ} \mathrm{C}$ ) for 5 and $24 \mathrm{hr}$, respectively. Filters were hybridized with a phosphoenolpyruvate carboxykinase (PEPCK) probe. The probe was labeled with $\left[{ }^{32} \mathrm{P}\right] \mathrm{dCTP}$ by the random priming method according to the manufacturer's instructions (Amersham). Blots were hybridized with the PEPCK probe and exposed to $\mathrm{x}$-ray film for 3 days at $-70^{\circ} \mathrm{C}$. The relative amounts of RNA loaded in each lane were visualized by staining the gel with ethidium bromide. Quantitation was performed by scanning densitometry of the $\mathrm{x}$-ray film using the NIH $\mathbf{1 . 6 2}$ program on an Apple Macintosh computer.

\section{Data Presentation}

Unless specified otherwise, data are presented as representative results from at least three independent experiments.

RESULTS AND DISCUSSION. To determine if L. sativus seeds contained GPI-like molecules, we employed established protocols that have been used for the extraction of GPI from mammalian tissues and cultured cell lines (15). After two consecutive rounds of t.l.c., a lipid that comigrated with rat liver GPI between the phospholipid standards PtdCho and PtdOH was identified as a phospholipid based on the following considerations: (i) that it was able to be stained with $\mathrm{I}_{2}$ vapor, (ii) that it was able to be stained with Coomassie blue, and (iii) that it reacted positively with a molybdenum spray reagent which is specific for compounds containing phosphate groups (Fig. 1). In addition, we were able to stain the phospholipid with ninhydrin, a specific reagent that reacts with free amino groups and with orcinol/

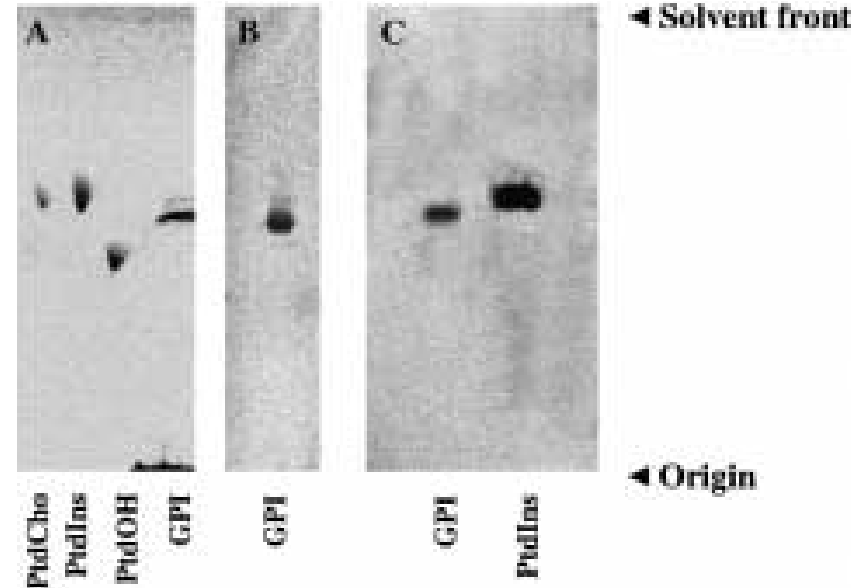

Fig. 1. Visualization of $L$. sativus seed GPI on t.l.c. plates. Purified GPI was applied to t.l.c. plates which were developed in the basic solvent system (see Materials and Methods).

(A) GPI stained with $I_{2}$ vapor. (B) GPI stained with Coomassie blue. (C) GPI stained with molybdenum blue reagent.

sulphuric acid, which reacts with sugar residues (results not shown). This multiple t.l.c. method has been proven to separate GPI from all neutral lipids and all other phospholipid classes (including the polyphosphoinositides, which may be contaminants after the first round of t.l.c.). Identical t.l.c. migration

Table 1. Fatty acid composition of L. sativus seed and rat liver GPI.

$\%$ of Total Fatty Acids

\begin{tabular}{lcc}
\cline { 2 - 3 } Fatty Acid & L. sativus GPI & Rat Liver GPI \\
\hline $14: 0$ & 2.5 & 3.8 \\
$15: 0$ & 1.3 & 1.3 \\
$16: 0$ & 34.6 & 53.3 \\
$17: 0$ & 1.3 & 1.9 \\
$18: 0$ & 35.7 & 37.2 \\
$18: 1 n-9$ & 3.5 & 2.5 \\
$18: 2 \mathrm{n}-6$ & 2.2 & ND \\
$22: 0$ & 4.0 & ND \\
$22: 1$ & 1.3 & ND \\
$23: 0$ & 3.0 & ND \\
$24: 0$ & 11.9 & ND \\
Saturated & 94.3 & 97.5 \\
Monounsaturated & 3.5 & 2.5 \\
Diunsaturated & 2.2 & ND
\end{tabular}

14:0, 14-carbon chain length fatty acid without a single carboncarbon double bond; $\mathrm{n}$, position of the first carbon-carbon double bond in the fatty acid.

ND, not detected. 
and chemical staining of rat liver GPI was observed when run in parallel (results not shown). Such t.l.c. behavior together with the positive staining led us to tentatively identify the spot as a putative GPI-like molecule. The sugar to phosphate to free amino group stoichiometry of the putative $L$. sativus seed GPI was estimated to be in a ratio of $3: 2: 1$. This indicated that the L. sativus seed GPI was less phosphorylated than rat liver GPI (15).

To further characterize the structure of the putative L. sativus seed GPI, we decided to determine its fatty acid composition. Transmethylation of the fatty acids converted them into their corresponding methyl esters. GC/MS revealed that the major fatty acids were palmitic (16:0), stearic (18:0), and lignoceric (24:4) acids together making up approximately $82 \%$ of total. Other fatty acids (containing saturated, monounsaturated, and diunsaturated structures) represented minor species (Table 1). When rat liver GPI was analyzed in parallel, a very limited set of fatty acid methyl esters were generated. In rat liver GPI, the major fatty acids were also saturated ones, 16:0 and 18:0 together making up approximately $90 \%$ of total. The only monounsaturated fatty acid found was oleic acid (18:1n-9) (2.5\% of total). Possible contamination of the putative L. sativus seed GPI or rat liver GPI with polyphosphoinositides was ruled out due to the fact that no polyunsaturated (e.g., arachidonic) fatty acid methyl esters were identified. The analysis indicated that in general terms the fatty acid composition of the putative L. sativus seed GPI was similar to that of rat liver GPI, suggesting some common biosynthetic and/or functional requirements.
One of the key features of GPI phospholipids is their susceptibility to hydrolysis by phospholipases $(15,18-20)$. Additional support for our claim that the putative L. sativus seed GPI was indeed a free GPI was strengthened by the finding that GPI-PLD, PI-PLC, and GPI-PLC caused the hydrolysis of the putative $L$. sativus seed GPI (Table 2). Of the three phospholipases used, GPI-PLD showed the greatest hydrolytic activity when the putative $L$. sativus seed GPI was incorporated into liposomes consisting of phosphatidylethanolamine (PtdEth), PtdCho, and cholesterol (Ch). The upper aqueous-methanolic phase formed after termination of the GPI hydrolysis incubation with GPI-PLD was lyophilized and both its phosphate and amino group content were estimated. This putative IPG showed a sugar to inorganic phosphate to amino group stoichiometry of 3:1:1. L. sativus seed GPI was a better substrate for the three enzymes than rat liver GPI (18). Together, our data on chemical composition and susceptibility to hydrolysis may suggest that seed GPI was a better substrate for phospholipases because it was less phosphorylated than rat liver GPI. Indeed, GPIspecific phospholipases and bacterial PI-PLC are specific for PI and GPI showing no or little activity toward PIPs (reviewed in 1,3).

To determine if the polar headgroup of GPI, the IPG, was generated as a consequence of the action of GPI-PLD on the seed GPI, we studied the biological activities corresponding to IPG. The inhibition of PKA activity in an in vitro assay has been the typical assay for the detection and assessment of the biological activity of IPG (21). We found that $5 \mu \mathrm{M}$ of IPG caused an inhibition (approximately $23 \%$ ) of PKA

Table 2. Hydrolysis of L. sativus seed GPI by phospholipases C and D.

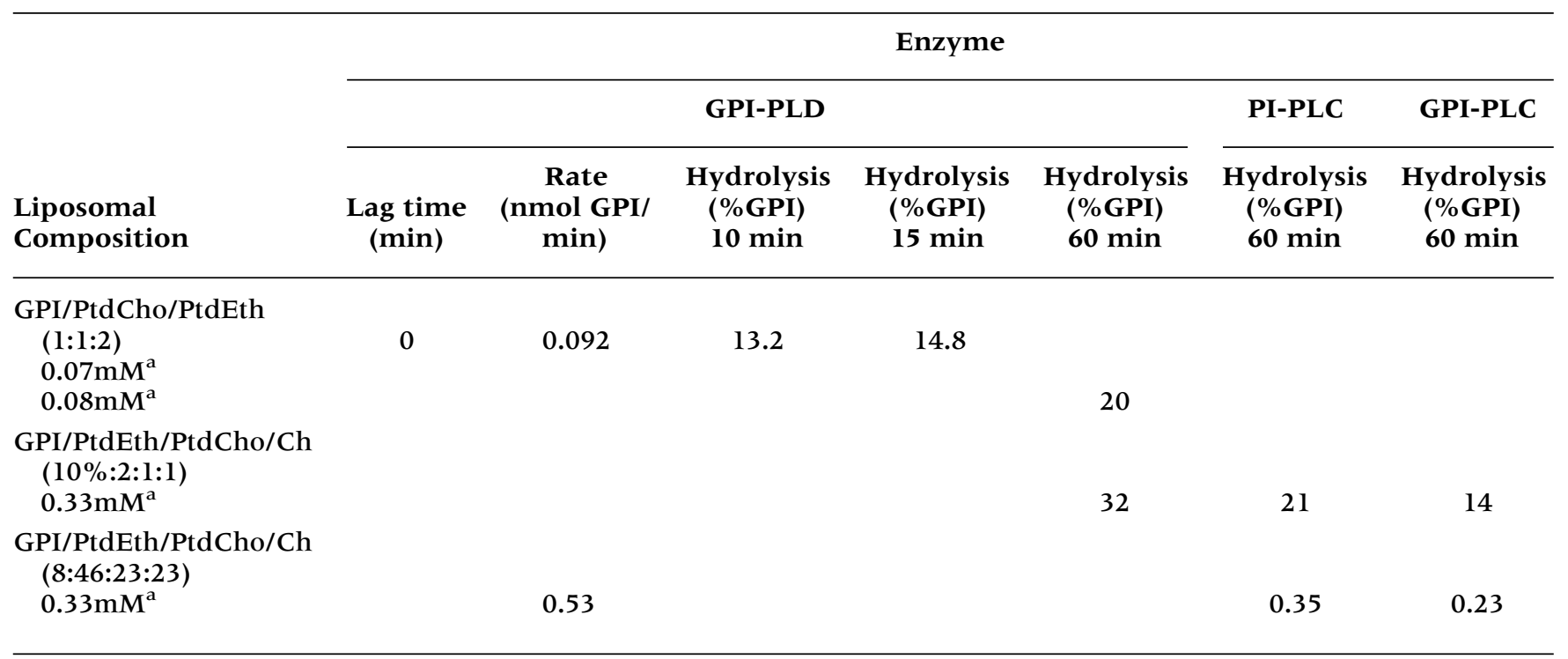

L. sativus seed GPI was reconstituted in liposomal membranes and then incubated with either GPI-PLD, GPI-PLC, or PI-PLC. ${ }^{a}$ All liposome preparations contained $13 \mu \mathrm{g}$ GPI at the start. The SEM showed $<10 \%$ variation. 
activity, similar to that seen with $10 \mu \mathrm{M}$ of rIPG prepared in an identical manner. When the concentration of IPG was increased to $10 \mu \mathrm{M}$, the observed inhibition was over $50 \%$ (Fig. 2A). These initial results indicate that biologically active IPG was generated after GPI-PLD-mediated hydrolysis of the putative $L$. sativus seed GPI.

Previous work from our laboratory demonstrated that the developing inner ear of the chicken embryo is a suitable model to study growth factorregulated proliferation and differentiation. IGF-I promotes growth, survival, and neurogenesis in both the otic vesicle and the CVG, which form part of the developing inner ear, in part through the GPI/ IPG signaling system $(23,26,29,30)$. In organotypic cultures of CVGs, IPG was able to increase the size of the CVG by almost 3-fold (Fig. 2B). When an identical concentration of rIPG was used in a parallel

A

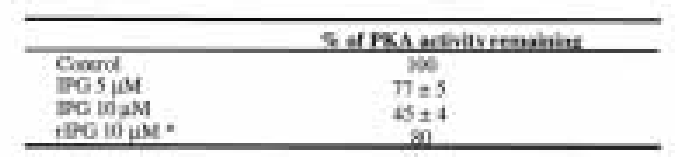

B

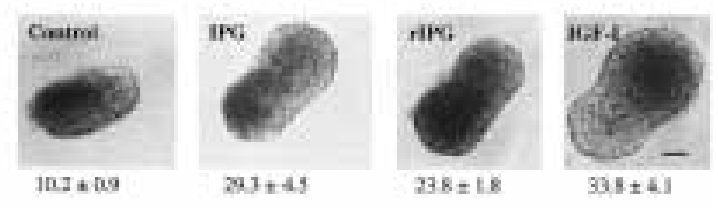

C

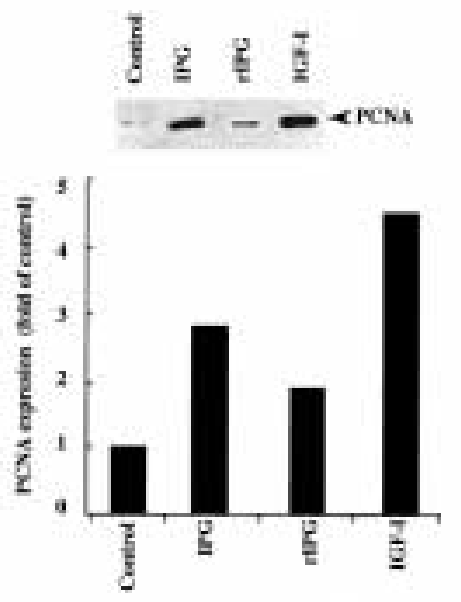

Fig. 2. Biological effects of the L. sativus seed IPG. (A) The ability of IPG and rIPG to inhibit the activity of PKA in an in vitro assay. *Taken from Villalba et al. (21). (B) The effect of no additions, IPG $(10 \mu \mathrm{M})$, rIPG $(10 \mu \mathrm{M})$ or IGF-I $(1 \mathrm{nM})$ on the growth of organotypic cultures of CVG. The calibration bar represents $100 \mu \mathrm{m}$. The numbers below each photograph represent CVG volume measurements (arbitrary units). (C) Upper: Western blot detection of PCNA expression within the CVG after treatment with no additions, IPG $(10 \mu \mathrm{M})$, rIPG $(10 \mu \mathrm{M})$ and IGF-I (1 nM). Lower: Densitometric analysis of the band corresponding to PCNA. IPG was obtained by hydrolysis of L. sativus seed GPI with GPI-PLD. incubation, the size of the CVG increased more than 2-fold (Fig. 2B). As expected and previously reported, incubation of CVG with IGF-I resulted in growth of the CVG (over 3-fold) (Fig. 2B). Interestingly, the effect of IPG was almost as powerful as IGF-I in the promotion of growth of the CVG. To provide evidence that the growth of the CVG was accompanied by changes in molecular markers, the expression of the PCNA was monitored by Western blotting (24-26). In Figure 2C, the detection of PCNA is shown. Densitometric scanning of the bands corresponding to the PCNA revealed that its intensity correlated with the increases in volume of the CVG (Fig. 2B) after treatment with IPG, rIPG, and IGF-I. As expected, treatment of the CVG with IGF-I led to an increase in the expression of PCNA (over 4-fold), greater than that of IPG (nearly 3-fold) and rIPG (approximately 2-fold). Using both morphometric analysis of organotypic explants and Western blotting detection of the PCNA (Figs. 2B and $2 \mathrm{C}$, respectively), we found that $L$. sativus seed IPG was slightly more active than rIPG. We do not yet have a full explanation for this observation. However, it is most likely that structural differences within the glycan structure of the IPG exist that may determine their biological activities, as indicated by studies with synthetic analogs (31).

Of all the growth factors and hormones known to cause the generation of IPG, insulin has been by far the most well documented $(1,3,4)$. One of the key factors for the characterization of IPG molecules is their ability to mimic certain metabolic effects of insulin. One of these metabolic effects is the reversal of 8-Br-cAMP-elevated PEPCK mRNA expression $(27,32)$. We decided to test whether L. sativus IPG had insulin-mimetic properties by using this experimental model. When the 8-Br-cAMP-treated H35 hepatoma cell line was treated with insulin, a decrease (of approximately $70 \%$ ) in the level of PEPCK MRNA was observed (Fig. 3). When rIPG and IPG were used in place of insulin, a smaller decrease (approximately 35\%) in the level of PEPCK mRNA was observed in both cases. These results are in agreement with those previously published $(27,32)$, and suggest that IPG prepared from human liver, rat liver, and L. sativus seed show similar insulin-mimetic activity.

Homeopathic extracts and infusions of plant material have long been used to try to reduce diabetic symptoms. However, the validity and compositional analysis of these treatments remain to be ascertained. One example is the antihyperglycemic agent 3-O-methyl D-chiro-inositol (pinitol) (33). This compound has been suggested to form part of the structure of a bovine liver-derived IPG (34). Furthermore, pinitol has been found to be present in the leaves of Bougainvillea, a plant that has been used to treat diabetes mellitus in India (35) and in gymnosperms and angiosperms (36). The novel glycoside 1D-2-O- $\alpha$-D-galactopyranosyl-chiro-inositol 

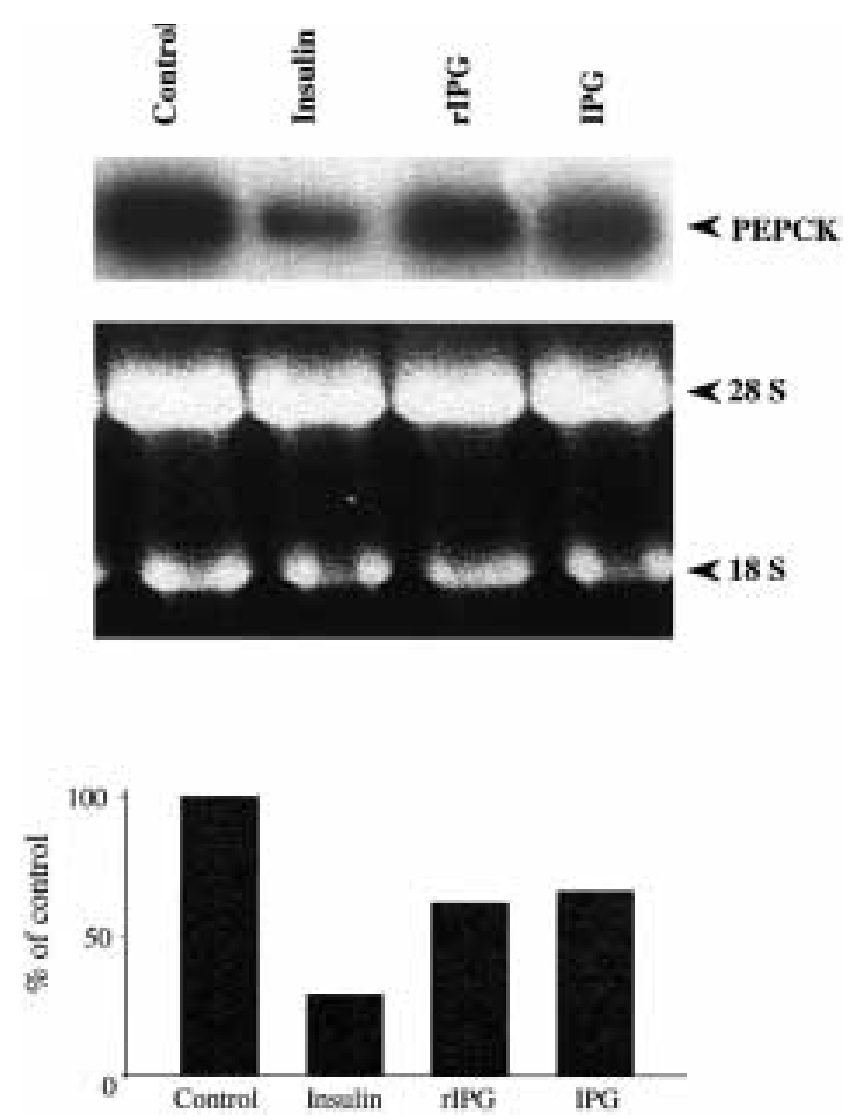

Fig. 3. Insulin-like effects of $L$. sativus IPG on PEPCK mRNA expression in $\mathbf{H} 35$ hepatoma cells. Serum-starved $\mathrm{H} 35$ cells were incubated in the presence of 8-Br-cAMP for 5 hours. Saline (lane 1), insulin (100 nM, lane 2), rIPG (10 $\mu \mathrm{M}$, lane 3$)$ or IPG (10 $\mu \mathrm{M}$, lane 4$)$ was added to the medium for the last 2 hours of 8-Br-cAMP treatment. PEPCK mRNA levels were analyzed by Northern blotting. An autoradiogram of a typical Northern blot is shown alongside the ethidium bromide-stained agarose gel from the same experiment. The Northern blot was scanned to allow quantification.

has been found in the beans of the jojoba plant Simmondsia chinensis (37). This compound shows some similarity to that of the structure of the proposed bovine liver-derived IPG. According to Fonteles et al. (33), the bovine liver-derived IPG contained a chelated transition metal. This was suggested to be manganese. In an extract of lucerne (Medicago sativa), used as an antihyperglycemic agent in South Africa, a high level of manganese was found (38). Whether this extract also contained IPG-like molecules still remains to be determined.

In summary, we have characterized a putative GPI phospholipid in L. sativus seeds that shows identical chromatographic and hydrolytic properties to those of GPI isolated from rat liver. Second, we have been able to demonstrate that the hydrolysis of the putative GPI phospholipid by GPI-PLD generates an IPG that shows in vitro inhibition of PKA activity and in vivo insulin-mimetic biological properties. Together, the results suggest that there are free GPI molecules in L. sativus seeds with properties very similar to those previously described in mammals. Further analyses of lipid and non-lipid extracts of L. sativus seeds will be necessary to substantiate whether they have antihyperglycemic properties. If such biochemical and pharmacologic criteria are met, then $L$. sativus seeds could be exploited for the purification of compounds, including IPG, which may help to design new agents to combat insulinresistant diseases.

\section{Acknowledgments}

The authors are grateful to Angélica Corral GarciaHeras (Finnova, Comunidad de Madrid) for excellent technical support and to Alberto Peluzzo and Lucía de la Rosa (C.R.F.-I.N.I.A.) for assistance with the L. sativus seeds. This work was partially supported by grants PM96-0075 and PB96-0171 (D.G.I.C.Y.T.), RF94-002 (C.R.F-I.N.I.A.) and 042.310G03/98 (U.P.V.). D.R.J. is a recipient of a research fellowship from the Comunidad de Madrid (research grant 08.3/0016.1/99). C.P. is a research fellow of Rademacher Group Ltd., London, UK. The Department of Immunology and Oncology was founded and is supported by the C.S.I.C. and Pharmacia and Upjohn.

\section{References}

1. Varela-Nieto I, León Y, Caro HN. (1996) Cell signaling by inositol phosphoglycans from different species. Comp. Biochem. Physiol. 115B: 223-241.

2. McConville MJ, Menon AK. (2000) Recent developments in cell biology and biochemistry of glycosylphosphatidylinositol lipids. Mol. Membr. Biol. 17: 1-16.

3. Jones DR, Varela-Nieto I. (1998) The role of glycosylphosphatidylinositol in signal transduction. Int. J. Biochem. Cell Biol. 30: 313-326.

4. Jones DR, Varela-Nieto I. (1999) Diabetes and the role of inositol-containing lipids in insulin signaling. Mol. Med. 5: 505-514.

5. Gray AM, Flatt PR. (1997) Pancreatic and extra-pancreatic effects of the traditional anti-diabetic plant, Medicago sativa (lucerne). Br. J. Nutr. 78: 325-334.

6. Fitzl G, Martin R, Dettmer D, Hermsdorf V, Drews H, Welt K. (1999) Protective effects of Gingko biloba extracts EGb 761 on myocardium of experimentally diabetic rats I: ultraestructural and biochemical investigation on cardiomyocytes. Exp. Toxicol. Pathol. 51: 189-198.

7. Welt K, Weiss J, Koch S, Fitzl G. (1999) Protective effects of Gingko biloba extracts EGb 761 on myocardium of experimentally diabetic rats II: ultraestructural and immunochemical investigation on microvessels and insterstitium. Exp. Toxicol. Pathol. 51: 213-222.

8. Vartanian GS, Karagezian KG. (1981) Normalizing effects of Bryonia alba $L$. On blood phospholipids in alloxan diabetes. Vopr. Med. Kim. 27: 179-181.

9. Karagezian KG, Vartanian GS, Panosian AG. (1981) Effect of an extract from the roots of bryony (Bryonia alba) on lipid peroxidation in the liver of rats with alloxan diabetes. Bull. Eksp. Biol. Med. 92: 35-37.

10. Almeida RN, Filho JM, Naik SR. (1985) Glycaemic and pharmacology of an ethanol extract of Bumelia sartorum. J. Ethnopharmacol. 14: 173-185.

11. Swanston-Flatt SK, Day C, Flatt PR, Gould BJ, Bailey CJ. (1989) Glycaemic effects of traditional European plant 
treatments for diabetes. Studies in normal and streptozotocin diabetic mice. Diabetes Res. 10: 69-72.

12. Swanston-Flatt SK, Day C, Bailey CJ, Flatt PR. (1990) Traditional plant treatments for diabetes. Studies in normal and streptozotocin diabetic mice. Diabetologia 33: 462-464.

13. Records of the C.R.F-I.N.I.A., Madrid, Spain.

14. Hoener MC, Brodbeck U. (1992) Phosphatidylinositol-glycanspecific phospholipase D is an amphiphilic glycoprotein that in serum is associated with high-density lipoproteins. Eur. $J$. Biochem. 206: 747-757.

15. Varela-Nieto I, Alvarez L, Mato JM. (1993) Intracellular mediators of peptide hormone action: glycosylphosphatidylinositol/inositol phosphoglycan system. In: de Pablo F, Scanes CG, eds. Handbook of Endocrine Research Techniques. New York: Academic Press, pp. 391-405.

16. Dietrich H, Espinosa JE, Chiara JL, et al. (1999) Glycosyl inositol derivatives related to inositolphosphoglycan mediators: synthesis, structure and biological activity. Chem. Eur. J. 5: $320-336$.

17. Martin-Lomas M, Khiar N, Garcia S, Koessler JL, Nieto PM, Rademacher TW. (2000) Inositolphosphoglycan mediators structurally related to glycosyl phosphatidylinositol anchors: synthesis, structure and biological activity. Chemistry 6: 3608-362 1 .

18. Villar AV, Goñi FM, Alonso A, Jones DR, León Y, VarelaNieto I. (1998) Phospholipase cleavage of glycosylphosphatidyl inositol reconstituted in liposomal membranes. FEBS Lett. 432: 150-154.

19. Villar AV, Alonso A, Pañeda C, Varela-Nieto I, Brodbeck U, Goñi FM. (1999) Towards the in vitro reconstitution of caveolae. Asymmetric incorporation of glycosylphosphatidylinositol (GPI) and gangliosides into liposomal membranes. FEBS Lett. 457: 71-74.

20. Jones DR, Avila MA, Sanz C, Varela-Nieto I. (1997) Glycosylphosphatidylinositol-phospholipase type D: a possible candidate for generation of second messengers. Biochem. Biophys. Res. Commun. 233: 432-437.

21. Villalba M, Kelly KL, Mato JM. (1988) Inhibition of cyclic AMP-dependent protein kinase by the polar head of an insulinsensitive glycophospholipid. Biochim. Biophys. Acta 968: 69-76.

22. Bernd P, Represa J. (1989) Characterization and localization of nerve growth factor receptors in the embryonic otic vesicle and cochleovestibular ganglion. Dev. Biol. 134: 11-20.

23. Represa J, Miner C, Giraldez F, et al. (1991) Glycosylphosphatidyl inositol phosphoglycans: a signaling system for the low-affinity nerve growth factor receptor. Proc. Natl. Acad. Sci. U.S.A. 88: 8016-8019.

24. Bravo R, MacDonald-Bravo H. (1987) Existence of two populations of cyclin/proliferating cell nuclear antigen during the cell cycle: association with DNA replication sites. J. Cell Biol. 105: 1549-1554.
25. Dietrich DR. (1993) Toxicological and pathological applications of proliferating cell nuclear antigen (PCNA), a novel endogenous marker for cell proliferation. Crit. Rev. Toxicol. 23: 77-109.

26. León Y, Sanz C, Giraldez F, Varela-Nieto I. (1998) Induction of cell growth by insulin and insulin-like growth factor-I is associated with jun expression in the otic vesicle. J. Comp. Neurol. 398: 323-332.

27. Alvarez L, Avila MA, Mato JM, Castaño JG, Varela-Nieto I. (1991) Insulin-like effects of inositol phosphate-glycan on messenger RNA expression in rat hepatocytes. Mol. Endocrinol. 5: 1062-1068.

28. Chomczynski P, Sacchi N. (1987) Single-step method of RNA isolation by acid guanidinium thiocianate-phenol-chloroform extraction. Ann. Biochem. 162: 156-159.

29. León Y, Vazquez E, Sanz C, et al. (1995) Insulin-like growth factor-I regulates the proliferation in the developing inner ear, activating glycosyl-phosphatidylinositol hydrolysis and fos expression. Endocrinology 136: 3494-3503.

30. León Y, Sanz C, Frago LM, et al. (1999) Involvement of insulin-like growth factor-I in inner ear organogenesis and regeneration. Horm. Metab. Res. 31: 126-132.

31. Frick W, Bauer A, Bauer J, Wied S, Müller G. (1998) Structure-activity relationship of synthetic phosphoinositolglycans mimicking metabolic insulin action. Biochemistry 37: 13421-13436.

32. Caro HN, Kunjara S, Rademacher TW, et al. (1997) Isolation and partial characterization of insulin-mimetic inositol phosphoglycans from human liver. Biochem. Mol. Med. 61: 214228.

33. Fonteles MC, Almeida MQ, Larner J. (2000) Antihyperglycemic effect of 3-O-methyl-D-chiro-inositol associatedwith manganese in streptozotocin diabetic rats. Horm. Metab. Res. 32: 129-132.

34. Fonteles MC, Huang LC, Larner J. (1996) Infusion of pH 2.0 Dchiroinositol glycan insulin putative mediator normalizes plasma glucose in streptozotocin diabetic rats at a dose equivalent to insulin without inducing hypoglycemia. Diabetologia 39: $731-734$.

35. Narayanan CR, Joshi JJ, Mujumdar AM, Dhekne VV. (1987) Pinitol: a new anti-diabetic compound from the leaves of Bougainvillea spectabilis. Curr. Sci. 56: 139-141.

36. Talbot HW Jr, Seidler RJ. (1979) Gas chromatographic analysis of in situ cyclitol utilization by Klebsielleae growing in redwood extracts. Appl. Environ. Microbiol. 38: 599-605.

37. Ogawa K, Watanabe T, Ikeda Y, Kondo S. (1997) A new glycoside 1D-2-O- $\alpha$-D galactopyranosyl- chiro-inositol from jojoba beans. Carbohyd. Res. 302: 219-221.

38. Rubenstein AH, Levin NW, Elliott GA. (1962) Manganeseinduced hypoglycaemia. Lancet II: 1348-1351. 